\title{
Methodology for Aligning and Comparing Spaceborne Radar and Ground-Based Radar Observations
}

\author{
Steven M. Bolen AND V. ChandRASEKAR \\ Colorado State University, Fort Collins, Colorado
}

(Manuscript received in final form 26 August 2002)

\begin{abstract}
Direct intercomparisons between space- and ground-based radar measurements can be a challenging task. Differences in viewing aspects between space and earth observations, propagation paths, frequencies, resolution volume size, and time synchronization mismatch between space- and ground-based observations can contribute to direct point-by-point intercomparison errors. This problem is further complicated by geometric distortions induced upon the space-based observations caused by the movements and attitude perturbations of the spacecraft itself. A method to align measurements between these two systems is presented. The method makes use of variable resolution volume matching between the two systems and presents a technique to minimize the effects of potential geometric distortion in space radar observations relative to ground measurements. Applications of the method are shown that make a comparison between the Tropical Rainfall Measuring Mission (TRMM) precipitation radar $(\mathrm{PR})$ reflectivity measurements and ground radar.
\end{abstract}

\section{Introduction}

A method has been developed that aligns space radar (SR) data with ground radar (GR) observations for the purpose of cross validation of the two systems. Accurate and precise alignment of observations requires meticulous attention to detail that is important in minimizing measurement uncertainties between the two systems so that quantitative analysis of bias, attenuation, and characterization of the microphysical properties of the atmospheric medium can be made along space radar beams via ground-based measurements. Reconciliation of the difference in resolution volumes between the two systems, due to size and orientation, and the need to align ground and space radar points have been previously investigated: Bolen and Chandrasekar (1999, 2000a) used a correlation-shift procedure and a momentbased technique, respectively, for final alignment of points. The work of Bolen and Chandrasekar was motivated by the need to evaluate attenuation correction in space radar echo returns. An independent procedure, motivated by the need to match reflectivity, was presented by Anagnastou et al. (2001), who provided a comprehensive study comparing space radar with ground radar reflectivity measurements. The method presented in this paper uses a variable volume matching scheme with a polynomial technique for alignment of SR and GR points. A theoretical modeling of potential

Corresponding author address: Dr. Steven M. Bolen NASA/JSC, MC:EV41, 2101 NASA Rd. 1, Houston, TX 77058.

E-mail: sbolen@ems.jsc.nasa.gov alignment errors is first performed to determine the expected magnitude of the errors and to analyze their impact on the intercomparison. Then the alignment methodology is demonstrated using data collected from the Tropical Rainfall Measuring Mission (TRMM) precipitation radar (PR) and S-band polarimetric (SPOL) radars on 13 August 1998. The procedure is also applied to simultaneous datasets taken between TRMM PR and the Kwajalein K-band polarization (KPOL) radars on 10 July 2000 as further illustrations of the alignment method.

Intercomparisons between ground radar and spaceborne radar on a point-by-point basis can be a difficult and challenging task. Errors result from the mismatch between GR and SR resolution volume, spatial image alignment, and operating frequencies. Differences in viewing aspects and resolution contribute to the intercomparison error, which results from the measurement of return signals from different volumes of the precipitation medium, as illustrated Fig. 1. Intercomparison is further complicated by geometric distortion introduced into the satellite data (e.g., shift, scale, shear, rotation, etc.), which is caused by the movements and attitude perturbations of the satellite itself (Schowengerdt 1997). Attitude motions such as roll, pitch, and yaw, as depicted in Fig. 2a, introduce variabilities into the retrieved satellite radar image that are not systematic in nature creating spatial alignment error between SR and GR images. Changes in the satellite altitude and velocity, due to orbit eccentricity, can also produce geometric distortion, as shown in Fig. 2b. Differences in operating 


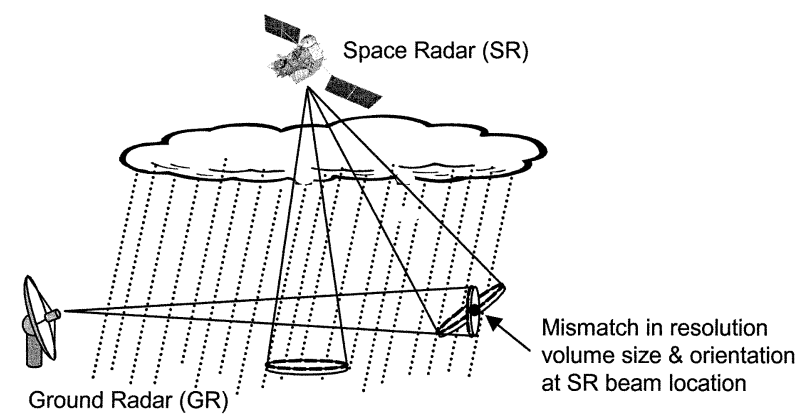

FIG. 1. Illustration of the viewing geometry for ground-based and spaceborne radar systems.

frequencies can also make it difficult to find alignment points (i.e., common reference points between the two radar images). Furthermore, attenuation and non-Rayleigh scattering of high-frequency space-based measurements (Bolen and Chandrasekar 2000b) can make the task of finding common points between return signals problematic in high-intensity storm regions. This paper presents a methodology for intercomparison that minimizes these errors.

\section{Alignment methodology}

To minimize errors, ground- and space-based data are collected during simultaneous intervals not exceeding 2-3 min in time difference. Small windows approximately $50 \mathrm{~km} \times 50 \mathrm{~km}$ in size (in a horizontal plane) are defined around a precipitation cell of interest so as to minimize nonlinear spatial geometric distortion effects. Both ground- and space-based data are remapped to a satellite-centered Cartesian coordinate system using a nonspherical earth model (WGS-84 model). For a cross-track, line scanning system, such as that employed by the TRMM PR, the coordinate system origin is located at the intersection of the satellite ground track and scan line that passes through the center of the storm area. The resample angle is taken such that the spaceborne radar line of scan becomes orientated perpendicular to the $x$ axis of the resampled coordinate grid. Both space- and ground-based data are sampled to grids spaced $0.5 \mathrm{~km}$ horizontally and $0.25 \mathrm{~km}$ in altitude. A 4/3-earth radius model is used during sampling of the ground radar data to compensate for radar beam refraction. The coordinate resampling scheme is illustrated in Fig. 3 showing ground radar data collected during the TEFLUN-B campaign on 13 August 1998.

Common reference points (or point pairs) are then found between the ground and space radar datasets. The procedure begins by finding the $(x, y)$ coordinates of each SR beam location, derived from satellite ephemeris and PR pointing data, in a horizontal plane at a nominal altitude. A three-dimensional set of reflectivity points is averaged in linear scale (units $\mathrm{mm}^{6} \mathrm{~m}^{-3}$ ) at each of the beam locations in both the horizontal and vertical
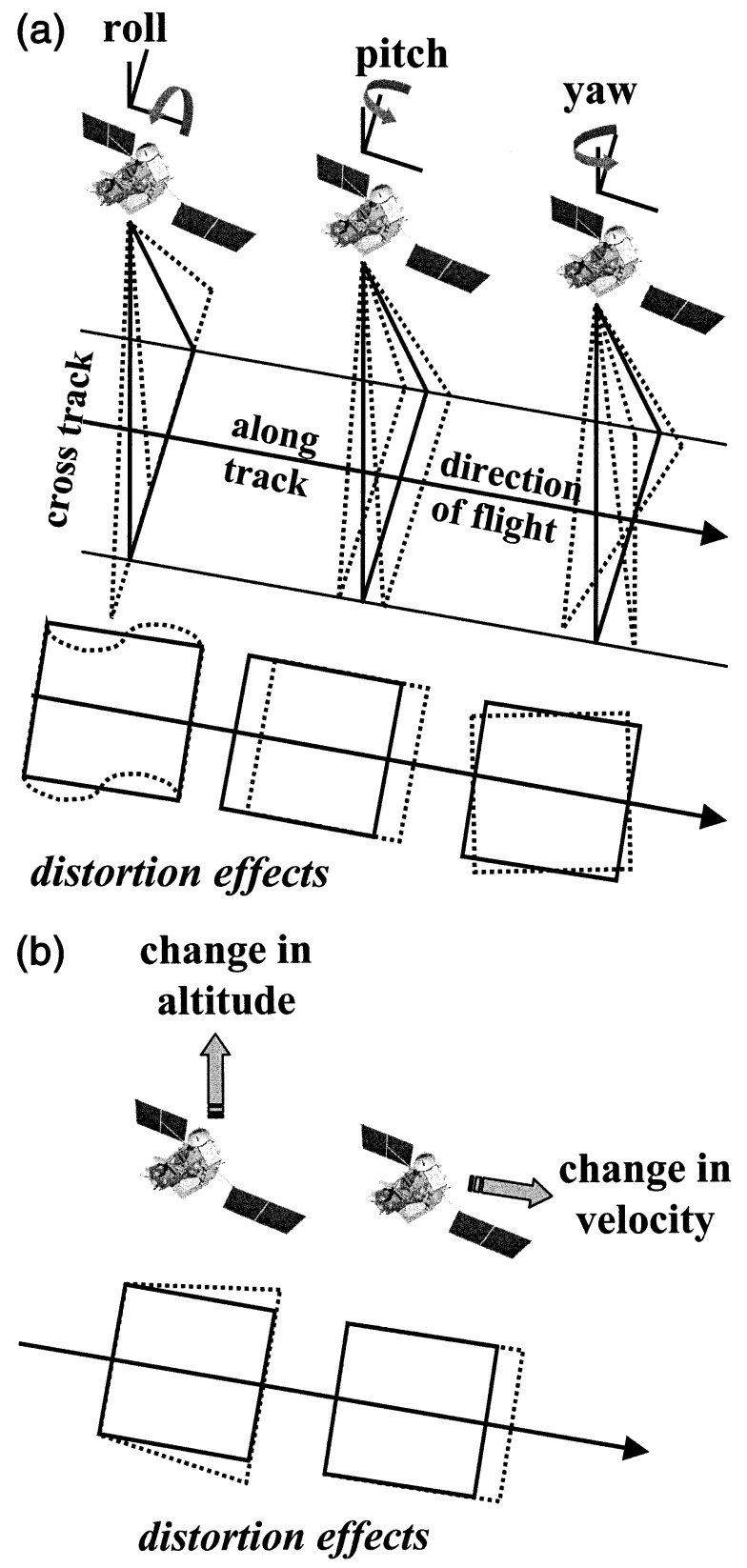

FIG. 2. Illustration of the types of platform motion that can cause geometric distortion in the space-based radar image. (a) Types of distortion caused by the satellite attitude perturbations: roll, pitch, and yaw. (b) Types of distortion caused by the satellite motions along its flight track.

directions in order to match the resolution volumes. The horizontal and vertical limits are taken as the maximum extent of either the SR or ground radar resolution at the SR beam location, which is calculated according to the geometry described in (Meneghini and Kozu 1990). The average value of reflectivity computed in the volume is taken as the satellite measured reflectivity, $Z_{m}(\mathrm{SR})$, at 


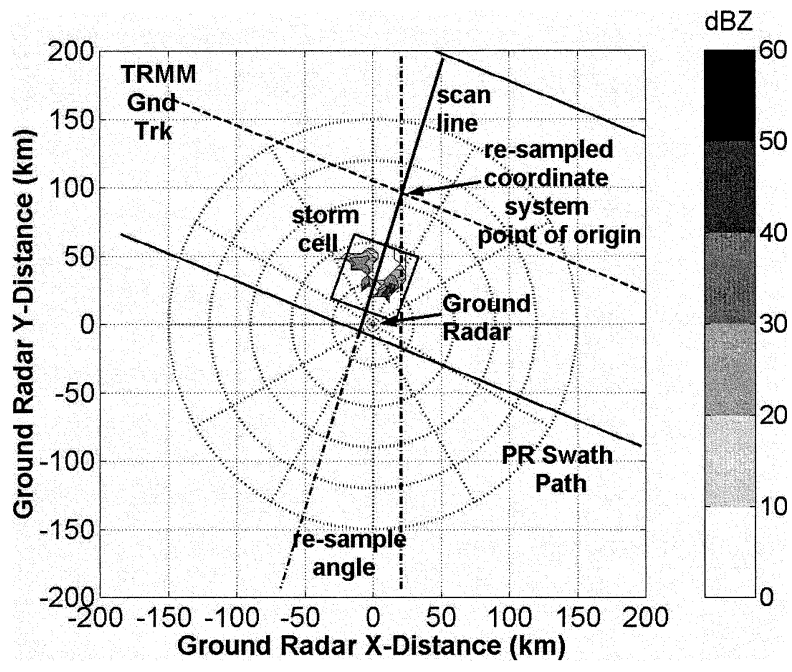

FIG. 3. Radar data remapping to space radar centered Cartesian coordinate system.

that $(x, y)$ location. Next, the points that comprise the volume are translated in $x, y$, and $z$ (i.e., along both horizontal directions and in the vertical direction) in the ground radar reflectivity dataset, starting from the SR beam location, $(x, y)$. The value computed is taken as the ground radar measured reflectivity, $Z_{m}(\mathrm{GR})$, at location $(x, y)$. The three-dimensional volume and illustration of the shift are shown in Fig. 4.

A ground-space radar point-pair is established when a shift in $(x, y, z)$ is found that minimizes a cost function based on the volume-averaged space and ground radar reflectivities, $Z_{m}(\mathrm{SR})$ and $Z_{m}(\mathrm{GR})$, and the Euclidean distance of the volume shift. Since it is assumed that the effect of attenuation is not significant on either radar at low-reflectivity contour levels at the edges of the storm cell, only volumes that result in GR reflectivities less than $30 \mathrm{dBZ}$ are considered as possible candidates for establishing a point-pair relationship. The cost function $(\mathrm{CF})$ is normalized based on the expected error in reflectivity measurements and PR beam location measurements, and is given by

$$
\begin{aligned}
\mathrm{CF}= & \frac{\left(\Delta x^{2}+\Delta y^{2}+\Delta z^{2}\right)^{1 / 2}}{\sigma_{d}} \\
& +\left|\frac{Z_{m}(\mathrm{PR})-Z_{m}(\mathrm{GR})+\text { bias }}{\sigma_{m}}\right|,
\end{aligned}
$$

where $\Delta x, \Delta y$, and $\Delta z$ are the incremental volume shift distances, $\sigma_{d}=\left(\sigma_{x}^{2}+\sigma_{y}^{2}+\sigma_{z}^{2}\right)^{1 / 2}$ is the expected error associated with the satellite geolocation and beampointing errors (derived from nominal satellite operational position vector and attitude errors in the horizontal and vertical directions denoted by $\sigma_{x}, \sigma_{y}$, and $\sigma_{z}$ ), and $\sigma_{m}$ is the reflectivity measurement uncertainty

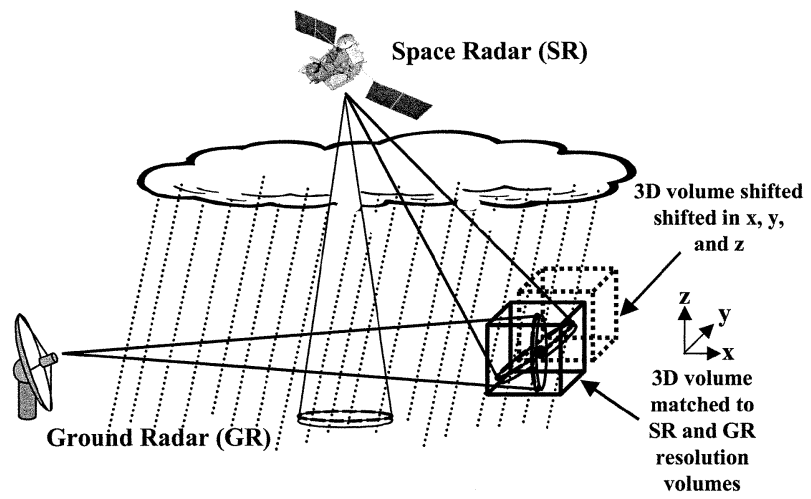

FIG. 4. Illustration of 3D volume matching and shifting based on GR and SR resolution volumes. The location of the SR beam is found at a nominal altitude, shown here at the 2-km altitude.

of the two systems. Amount of computation effort is limited by considering only shifts within the distance of $2 \sigma_{d}$. Finally, in order to minimize any potential calibration bias between space and ground radar measurements, a bias term is introduced into the second term on the right-hand side of Eq. (1). The bias is determined by taking the difference of the arithmetic mean between the space and ground radar reflectivities over a three-dimensional volume with dimensions of $\sigma_{x}, \sigma_{y}$, and $\sigma_{z}$ centered about the SR beam location.

Once the set of point pairs is found, a polynomial fit is used to determine the appropriate mapping of the space radar image to the ground radar image. Following the development of Schowengerdt (1997), a polynomial of order $N$ can be used to relate the coordinates of the contour of the distorted spaceborne image $\left[x_{c}(\mathrm{SR})\right.$, $\left.y_{c}(\mathrm{SR})\right]$ to the corresponding ground radar contour coordinates $\left[x_{c}(\mathrm{GR}), y_{c}(\mathrm{GR})\right]$ via

$$
\begin{aligned}
& x_{c}(\mathrm{GR})=\sum_{i=0}^{N} \sum_{j=0}^{N-i} \alpha_{i j} x_{c}(\mathrm{SR})^{i} y_{c}(\mathrm{SR})^{i} \\
& y_{c}(\mathrm{GR})=\sum_{i=0}^{N} \sum_{j=0}^{N-i} \beta_{i j} x_{c}(\mathrm{SR})^{i} y_{c}(\mathrm{SR})^{i} .
\end{aligned}
$$

The coefficients, $\alpha$ and $\beta$, are determined and applied to the entire SR dataset, thereby mapping the space radar image to the ground radar image. The level of detail that can be approximated in the fit depends on the order of the polynomial. Usually, a quadratic polynomial in $x$ and $y$ (i.e., six coefficients) is sufficient for most satellite remote sensing applications (Schowengerdt 1997). This type of transformation method can be used to reduce the effects of shift, scale, shear, rotation, etc. between the two images. For the set of SR data coordinate locations $[x(\mathrm{SR}), y(\mathrm{SR})]$ and GR data locations $[x(\mathrm{GR})$, $y(\mathrm{GR})]$, Eq. (2) can be written in the general vectormatrix form, for six coefficients and $m$ point-pairs, as 


$$
\left[\begin{array}{c}
x(\mathrm{GR})_{1} \\
x(\mathrm{GR})_{2} \\
\vdots \\
x(\mathrm{GR})_{m}
\end{array}\right]=\left[\begin{array}{cccccc}
1 & x(\mathrm{SR})_{1} & y(\mathrm{SR})_{1} & x(\mathrm{SR})_{1} y(\mathrm{SR})_{1} & x(\mathrm{SR})_{1}^{2} & y(\mathrm{SR})_{1}^{2} \\
1 & x(\mathrm{SR})_{2} & y(\mathrm{SR})_{2} & x(\mathrm{SR})_{2} y(\mathrm{SR})_{2} & x(\mathrm{SR})_{2}^{2} & y(\mathrm{SR})_{2}^{2} \\
\vdots & \vdots & \vdots & \vdots & \vdots & \vdots \\
1 & x(\mathrm{SR})_{m} & y(\mathrm{SR})_{m} & x(\mathrm{SR})_{m} y(\mathrm{SR})_{m} & x(\mathrm{SR})_{m}^{2} & y(\mathrm{SR})_{m}^{2}
\end{array}\right]\left[\begin{array}{c}
\alpha_{00} \\
\alpha_{10} \\
\alpha_{01} \\
\alpha_{11} \\
\alpha_{20} \\
\alpha_{02}
\end{array}\right],
$$

or, in more compact form as

$$
\mathbf{X}(\mathrm{GR})=\mathbf{W A},
$$

where bold-face serif represents a vector, and bold-face sans serif represents a matrix, form with $\mathbf{X}(\mathrm{GR})$ being the vector of GR $x$ coordinates, $\mathbf{W}$ being the matrix with structure shown in the first product on the right-handside of Eq. (4) and $\mathbf{A}$ being the vector of $\alpha$ coefficients that map SR observation locations to GR $x$ coordinate locations. The mapping of SR coordinates to GR $y$ coordinates in Eq. (3) can be written in vector-matrix form similar to Eq. (4), and also in the more compact form

$$
\mathbf{Y}(\mathrm{GR})=\mathbf{W B},
$$

with $\mathbf{Y}(\mathrm{GR})$ being the vector of GR $y$ coordinates, $\mathbf{W}$ as described before for Eq. (5), and $\mathbf{B}$ being the vector of $\beta$ coefficients that map SR observation locations to GR $y$-coordinate locations. Special physical interpretation can be placed on each of the coefficients contained in A and B as components to the total "warp," or geometric distortion, in the space radar image. A summary of the properties is listed in Table 1 (Schowengerdt 1997).

If the number of point-pairs is equal to the number of polynomial coefficients, (e.g., $m=6$ for a secondorder polynomial), the ideal error in the polynomial fit would be zero. However, since some point pairs may be in error (e.g., due to incorrect volume matching), it is desirable to find more pairs, such that the number of point-pairs is more than then number of coefficients in order to minimize the impact of incorrect matching. In this case, (5) and (6) can be written as

TABLE 1. Physical meaning of each coefficient to the total warp in the space radar image for the $x$ and $y$ coefficients (Schowengerdt 1997).

\begin{tabular}{cl}
\hline \hline Coefficient & \multicolumn{1}{c}{ Warp component } \\
\hline$\alpha_{00}$ & Shift in $x$ \\
$\beta_{00}$ & Shift in $y$ \\
$\alpha_{10}$ & Scale in $x$ \\
$\beta_{01}$ & Scale in $y$ \\
$\alpha_{01}$ & Shear in $x$ \\
$\beta_{10}$ & Shear in $y$ \\
$\alpha_{11}$ & $y$-dependent scale in $x$ \\
$\beta_{11}$ & -dependent scale in $y$ \\
$\alpha_{20}$ & Nonlinear scale in $x$ \\
$\beta_{02}$ & Nonlinear scale in $y$ \\
\hline
\end{tabular}

$$
\begin{aligned}
& \mathbf{X}(\mathrm{GR})=\mathbf{W} \mathbf{A}+\varepsilon_{\mathbf{x}} \\
& \mathbf{Y}(\mathrm{GR})=\mathbf{W B}+\varepsilon_{\mathbf{y}},
\end{aligned}
$$

where $\varepsilon_{\mathrm{x}}$ and $\varepsilon_{\mathrm{y}}$ represent the possible location errors between point pairs associated with GR $x$ and $y$ coordinates, respectively. Solutions to (7) and (8) can be found by minimizing the error term as

$$
\begin{aligned}
& \min \left[\varepsilon_{\mathbf{x}}^{\mathrm{T}} \boldsymbol{\varepsilon}_{\mathbf{x}}\right]=[\mathbf{X}(\mathrm{GR})-\mathbf{W} \hat{\mathbf{A}}]^{\mathrm{T}}[\mathbf{X}(\mathrm{GR})-\mathbf{W} \hat{\mathbf{A}}] \\
& \min \left[\varepsilon_{\mathbf{y}}^{\mathrm{T}} \boldsymbol{\varepsilon}_{\mathbf{y}}\right]=[\mathbf{Y}(\mathrm{GR})-\mathbf{W} \hat{\mathbf{B}}]^{\mathrm{T}}[\mathbf{Y}(\mathrm{GR})-\mathbf{W} \hat{\mathbf{B}}],
\end{aligned}
$$

where $\mathrm{T}$ represents transpose and $\hat{\mathbf{A}}$ and $\hat{\mathbf{B}}$ are the least squares solutions for Eqs. (9) and (10), which are given by

$$
\begin{aligned}
& \hat{\mathbf{A}}=\left(\mathbf{W}^{\mathrm{T}} \mathbf{W}\right)^{-1} \mathbf{W}^{\mathrm{T}} \mathbf{X}(\mathrm{GR}) \\
& \hat{\mathbf{B}}=\left(\mathbf{W}^{\mathrm{T}} \mathbf{W}\right)^{-1} \mathbf{W}^{\mathrm{T}} \mathbf{Y}(\mathrm{GR})
\end{aligned}
$$

A weighted least squares solution can be obtained that minimizes the error terms (Brogan 1985)

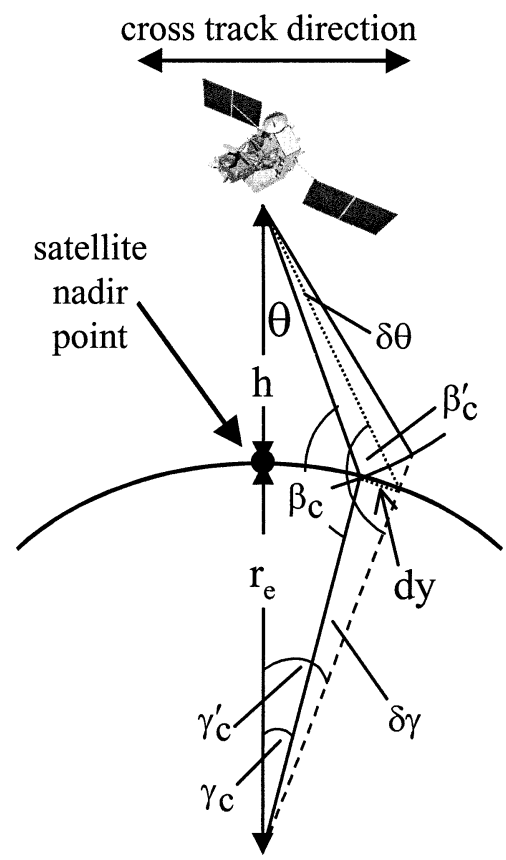

FIG. 5. Geometry for determining the magnitude of cross-track position perturbation due to attitude roll motion. 


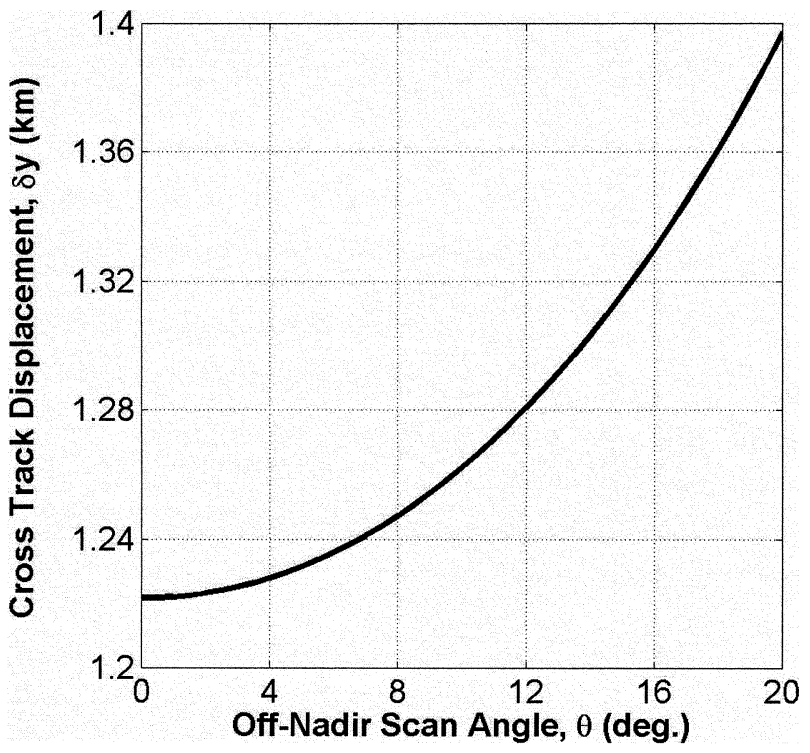

FIG. 6. Plot of potential cross track displacement caused by attitude roll motion assuming $0.2^{\circ}$ of attitude uncertainty.

$$
\begin{aligned}
& \min \left[\varepsilon_{\mathbf{x}}^{\mathrm{T}} \varepsilon_{\mathbf{x}}\right]=[\mathbf{X}(\mathrm{GR})-\mathbf{W} \hat{\mathbf{A}}]^{\mathrm{T}} \mathbf{Q}[\mathbf{X}(\mathrm{GR})-\mathbf{W} \hat{\mathbf{A}}] \\
& \min \left[\varepsilon_{\mathbf{y}}^{\mathrm{T}} \varepsilon_{\mathbf{y}}\right]=[\mathbf{Y}(\mathrm{GR})-\mathbf{W} \hat{\mathbf{B}}]^{\mathrm{T}} \mathbf{Q}[\mathbf{Y}(\mathrm{GR})-\mathbf{W} \hat{\mathbf{B}}] \\
& \text { with the solutions for } \hat{\mathbf{A}} \text { and } \hat{\mathbf{B}} \text { given by } \\
& \qquad \begin{array}{c}
\hat{\mathbf{A}}=\left(\mathbf{W}^{\mathrm{T}} \mathbf{Q} \mathbf{W}\right)^{-1} \mathbf{W}^{\mathrm{T}} \mathbf{Q X}(\mathrm{GR}) \\
\hat{\mathbf{B}}=\left(\mathbf{W}^{\mathrm{T}} \mathbf{Q} \mathbf{W}\right)^{-1} \mathbf{W}^{\mathrm{T}} \mathbf{Q Y}(\mathrm{GR}) .
\end{array}
\end{aligned}
$$

Here, $\mathbf{Q}$ is a nonsingular diagonal matrix such that its elements, $q_{i j}$, are related to the expected confidence in the $j$ th point-pair matching (for $i=j$, and equal to zero for $i \neq j$ ). That is to say, given the cost function values computed from Eq. (1), a relative measure of confidence can be determined corresponding to the validity in the establishment of a particular ground-space radar contour point-pair matching. It is assumed that point pairs that have a relatively low minimum cost function value are more closely matched than point pairs with higher $\mathrm{CF}$ values. Each element, $q_{i j}$, in $\mathbf{Q}$, is determined from the cost function in the following way:

$$
q_{i j}= \begin{cases}\max [\mathrm{CF}]-\mathrm{CF}_{j}+\min [\mathrm{CF}] & i=j \\ 0 & i \neq j,\end{cases}
$$

where $\mathrm{CF}_{j}$ is the cost function of the $j$ th pair. For the set of point pairs, the maximum and minimum cost functions are found and the elements, $q_{i j}$, of the $\mathbf{Q}$ matrix are determined according to Eq. (17). This ensures that pairs with smaller cost functions are weighted more than pairs with larger cost functions in the weighted least squares solution. This also ensures that no diagonal element of $\mathbf{Q}$ is equal to zero.

In practice, it may be possible that one or more point pairs could have cost function values that are the same, or nearly the same. Because of this, several point-pair sets could be reasonably established between the ground and space radar storm cell contours. For this reason, the difference in GR and SR volume matched reflectivity values are taken over the echo contours that lie in the $20-30-\mathrm{dBZ}$ range (where $20 \mathrm{dBZ}$ is about $3 \mathrm{~dB}$ over the PR's nominal sensitivity level). The set of point pairs that minimizes the standard deviation of the difference is taken as the valid point pair set. This approach minimizes the possibility of selecting the incorrect set of point pairs, and also has the effect of selecting a pointpair set that minimizes the scatter between GR and PR reflectivity observations. Finally, alignment in the vertical direction between space and ground radar measurements can also be made from $\Delta z$ : the shift in the vertical that minimizes the cost function.

\section{Effects of geometric distortion on SR data retrieval}

\section{a. Uncertainty in SR beam location}

Matching SR and GR resolution volumes to take into account variations in resolution size and orientation between the two systems is a straightforward notion. However, the necessity to account for geometric warping in SR data retrieval may seem less obvious. Satellite attitude perturbations, such as roll, pitch, and yaw, can produce displacement shifts or rotation in the SR return image. Uncertainties associated with these types of movements can potentially be significant leading to localized distortion in SR observations relative to GR measurements, most notably near the edges of the SR swath path. In principle, for a nadir-looking, scanning system, like the TRMM PR, the magnitude of the variations can be determined from the satellite ephemeris: satellite altitude, off-nadir scan and roll angle perturbation in the cross-track direction, and pitch angle perturbation in the along-track direction. As shown in Fig. 5 , for a given off-nadir scan angle $\theta$ with the satellite at nominal altitude $h$, the displacement $\delta y$ in the crosstrack direction due roll perturbation angle $\delta \theta$ can be determined as follows.

From the law of sines, the angle $\beta_{c}$, can be determined from $\sin \beta_{c} /\left(r_{e}+h\right)=\sin \theta / r_{e}$, where $r_{e}$ is the earth radius, which is

$$
\beta_{c}=\sin ^{-1}\left[\frac{\left(r_{e}+h\right)}{r_{e}} \sin \theta\right]
$$

The central angle $\gamma_{c}$, which subtends the arc from the satellite nadir point to the expected beam location at scan angle $\theta$, is $\gamma_{c}=90-\beta_{c}-\theta$. Likewise, the central angle with roll perturbation $\delta \theta$ can be determined from similar geometric analysis, which yields

$$
\beta_{c}^{\prime}=\sin ^{-1}\left[\frac{\left(r_{e}+h\right)}{r_{e}} \sin (\theta+\delta \theta)\right],
$$

from which it follows that $\gamma_{c}^{\prime}=90-\beta_{c}^{\prime}-\theta-\delta \theta$ 
(a)

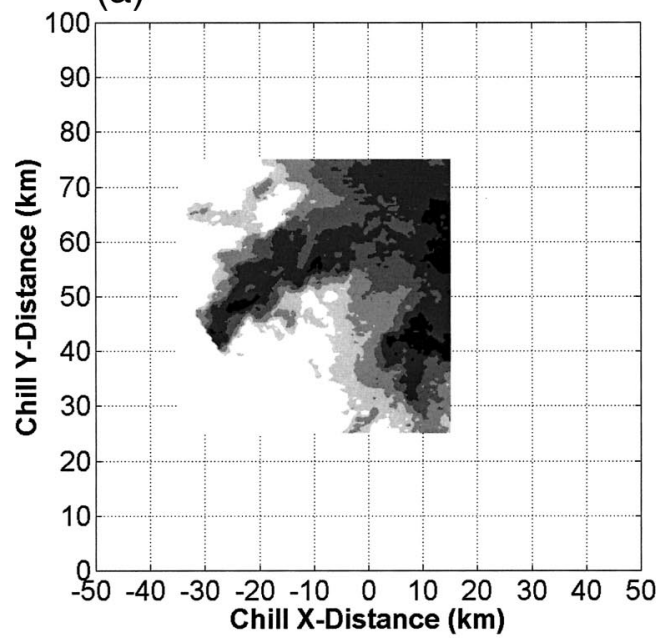

(c)

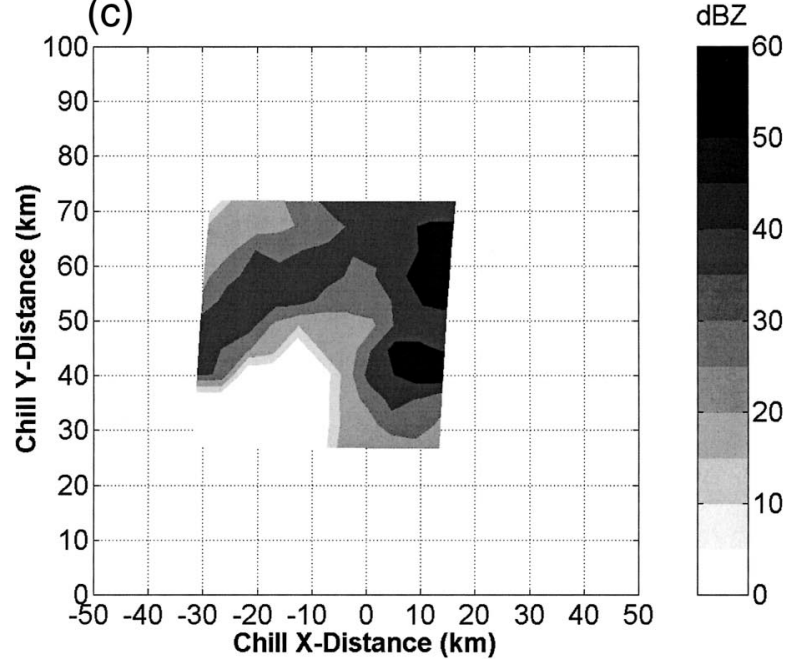

(where the prime denotes perturbation-induced quantities). The cross-track shift can then be found via

$$
\frac{\left(\frac{d y}{2}\right)}{r_{e}}=\sin \left(\frac{\delta \gamma_{c}}{2}\right),
$$

where $\delta \gamma_{c}=\gamma_{c}^{\prime}-\gamma_{c}$. Simplification yields the following result for the cross-track shift due to roll perturbation:

$$
d y=2 r_{e} \sin \left(\frac{\delta \gamma_{c}}{2}\right)
$$

Using Eqs. (18) and (19) in the expression for $\delta \gamma_{c}$, and substituting into Eq. (21), the following equation can be derived for the cross-track displacement due to roll perturbation:

$$
d y=2 r_{e} \sin \left(\frac{\beta_{c}-\beta_{c}^{\prime}-\delta \theta}{2}\right) .
$$

(b)

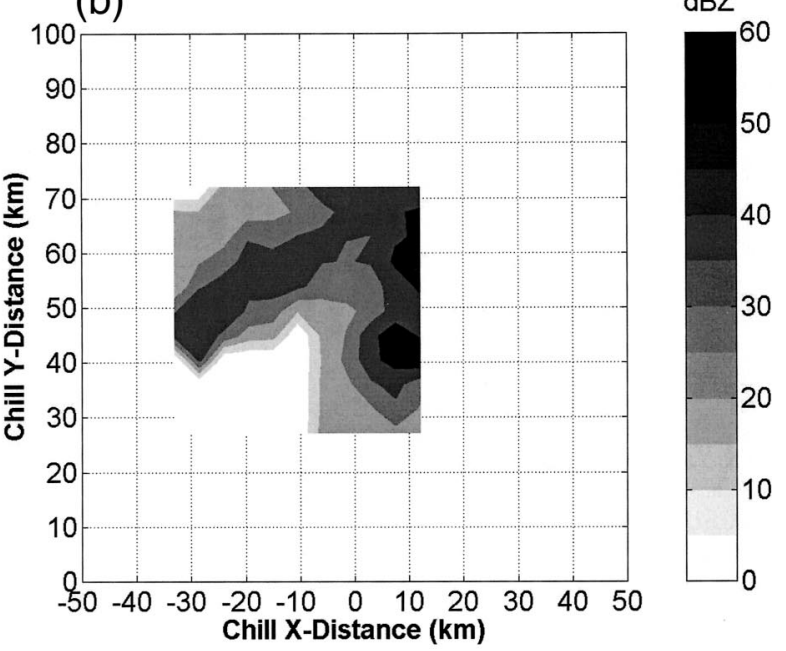

FIG. 7. Simulation of TRMM PR reflectivity using CHILL data taken from the STEPS campaign on 23 Jun 2000. (a) CHILL data image at $2-\mathrm{km}$ CAPPI before simulation shown at $0.5-\mathrm{km}$ horizontal resolution. (b) Simulated PR reflectivity image with resolution assumed to be $4 \mathrm{~km}$ in the horizontal and matched to CHILL beamwidth (c) PR reflectivity image of (b) with geometric warping introduce and $1 \sigma$ noise added.

The along-track shift $d x$ due to pitch angle perturbation $\delta \phi$ is determined in a similar fashion with the exception that there is no scan angle in the along-track direction. The along-track shift is

$$
d x=2 r_{e} \sin \left(\frac{\beta_{a}-\beta_{a}^{\prime}-\delta \phi}{2}\right),
$$

where $\beta_{a}$ has an analogous meaning as $\beta_{c}$, but in the along-track direction, and is given by

$$
\beta_{a}=\sin ^{-1}\left[\frac{\left(r_{e}+h\right)}{r_{e}}\right],
$$

and, likewise, the angle after perturbation is

$$
\beta_{a}^{\prime}=\sin ^{-1}\left[\frac{\left(r_{e}+h\right)}{r_{e}} \sin (\delta \phi)\right] .
$$

The potential cross-track displacement error for off-nadir scan angles between $0^{\circ}$ and $17^{\circ}$ with roll angle uncertainty $0.2^{\circ}$ is plotted in Fig. 6 for a satellite platform 
at a nominal altitude of $350 \mathrm{~km}$. Note that the magnitude of the displacement shift at scan angle $0^{\circ}$ is also the magnitude of the along-track shift if pitch angle uncertainty is also assumed to be $0.2^{\circ}$. As scan angle increases, the displacement due to attitude perturbation is also seen to increase. For the PR, with nominal 4.3-km resolution near the earth surface, displacement shift, due to roll perturbation, can be as large as about $1.34 \mathrm{~km}$ in cross-track and about $1.23 \mathrm{~km}$ in the along-track direction due to pitch perturbation (as indicated at the $0^{\circ}$ off-nadir scan).

Geometric distortion due to yaw perturbation effects with uncertainty angle $\delta \rho$ is of the form

$$
\begin{aligned}
& x^{\prime}=y \sin (\delta \rho) \\
& y^{\prime}=y \cos (\delta \rho),
\end{aligned}
$$

where $x$ and $y$ are the SR along-track and cross-track beam locations, respectively (with the prime indicating the coordinates due yaw uncertainty). For a yaw uncertainty of $0.2^{\circ}$, cross-track displacement is minimal. However, the along-track shift can be as large as $0.35 \mathrm{~km}$ near the SR swath edge at about $100 \mathrm{~km}$. Taking into account yaw and pitch perturbation, the total along-track shift could, therefore, be as large as $1.6 \mathrm{~km}$ with rotation accounting for nearly $20 \%$ of the displacement. Finally, satellites operating in noncircular orbits can undergo uncertainty variations in altitude and along-track velocity. These types of variations are the result of the nonsphericity of the earth and orbit eccentricity. Depending on the magnitude of the uncertainties, these can contribute to geometric warping, but are small for the PR.

\section{b. Geometric distortion effects}

High-resolution reflectivity measurements taken from the Colorado State University (CSU) CHILL radar during the STEPS campaign on 23 June 2000 were used to simulate TRMM PR observations in order to determine the magnitude of the effects of geometric distortion on SR data. A cross-sectional profile of the CHILL data at $2 \mathrm{~km}$ CAPPI (constant altitude planned position indicator) is shown in Fig. 7a sampled at $0.5-\mathrm{km}$ resolution. The CHILL reflectivity measurements $\left(Z_{h}\right)$ were degraded in resolution to simulate the effects of matching PR resolution volume to the ground radar resolution, as described in preceding sections. The resulting image is shown in Fig. 7b. A 1-dB zero mean Gaussian noise was added to the matched resolution data to simulate the measurement uncertainty in PR reflectivity data as described in the TRMM Science User Interface Control Specification (NASA 1999). The reflectivity field was then warped according to the polynomial coefficients: $\mathbf{A}=[0.8254000$ $\cos (0.0034970) \sin (0.0034970)-0.0000700 .000001$ $0.000650]^{\mathrm{T}}, \quad \mathbf{B}=[-0.284600-\sin (0.0034970)$ $\cos (0.0034970)-0.0000100 .0000000 .000000]^{\mathrm{T}}$, and $\mathbf{C}$ $=0.0$ (where $\mathbf{C}$ is the altitude uncertainty) to simulate geometric distortion in PR observations. Here, the distor-

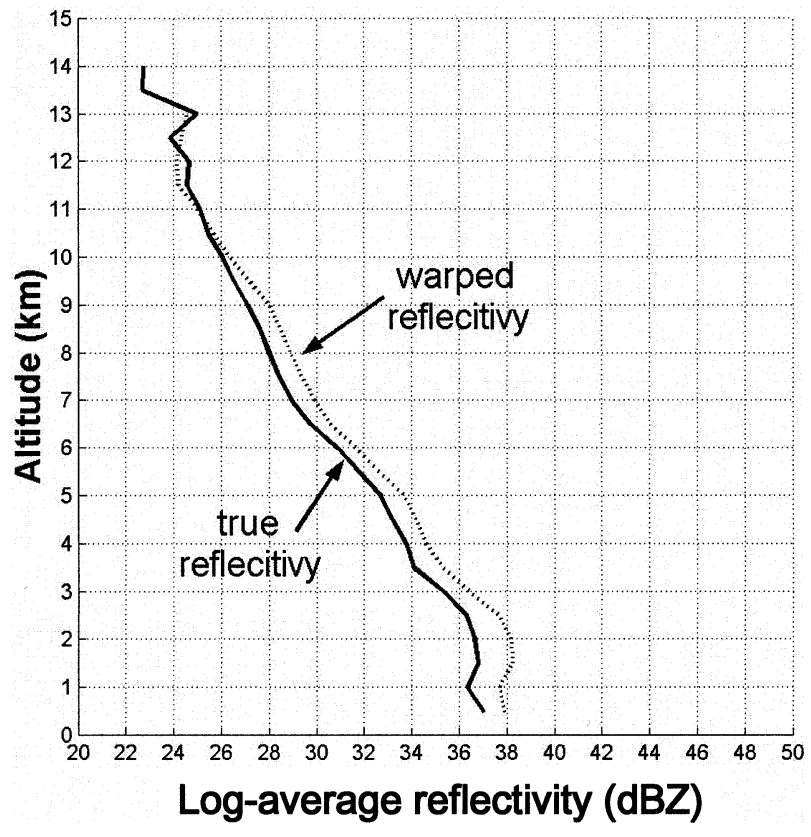

FIG. 8. Plot of average $Z_{h}$ vs altitude for both log-averaged and linear-averaged reflectivity from simulated PR data.

tion is primarily due to shift and rotation (about $0.2^{\circ}$ ) with very small amounts of other distortion. The distorted, or warped $Z_{h}$ field, is shown in Fig. 7c. The total area $\geq 20$ $\mathrm{dBZ}$ after warping is about $2.5 \%$ larger than the area before warping. That is, in this example, the warping increased the total area of the storm cell by about $2.5 \%$ using the $20-\mathrm{dBZ}$ contour as a measure. The reflectivity was averaged in $\log _{10}$ scale (henceforth referred to as log scale with subscript omitted) across horizontal planes for both the true and warped reflectivity fields at $0.5-\mathrm{km}$ vertical increments ranging from 0 to $15 \mathrm{~km}$. The averages were plotted with respect to altitude as shown in Fig. 8, where the true reflectivity is depicted with a solid line and the warped reflectivity with a dotted line. The difference between true and warped reflectivity is seen to be as much as $1.4 \mathrm{~dB}$.

From modeling, it is found that geometric warping, due to satellite motions, can affect the magnitude of the measured reflectivities. Geometric distortion is a nonlinear effect, which introduces nonsystematic variabilities into the retrieved SR image. This has an especially acute effect on nonhomogeneous radar observations. In this example, the radar image is spatially "warped" in a nonlinear way creating a distorted ratio of high-intensity area to low-intensity area. This is evident in comparison of Fig. 7b with Fig. 7c where the highintensity area in the upper-right corner of image has become exaggerated due to warping compared to other parts of the image. The effect of warping introduces a bias on average of $1.0 \mathrm{~dB}$ into the retrieved PR measurements for log-averaged reflectivity below $9 \mathrm{~km}$, as shown in Fig. 8. From modeling, it can perhaps be concluded that geometric distortion is a possibility, and that 
(a)

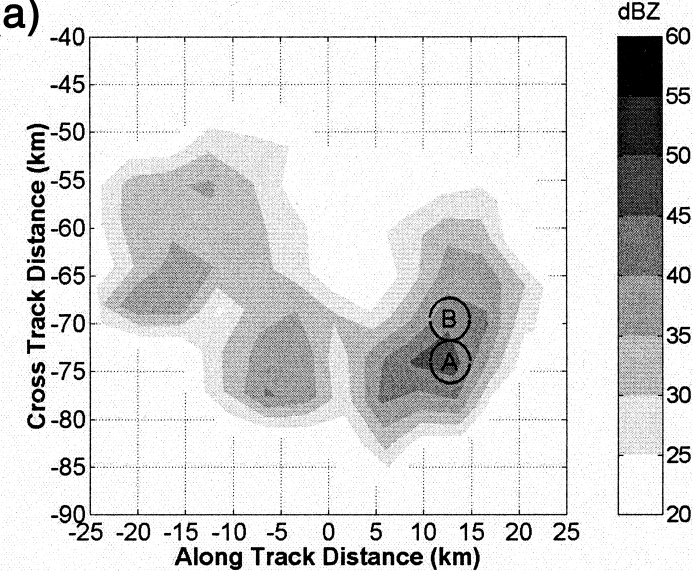

\section{(b)}

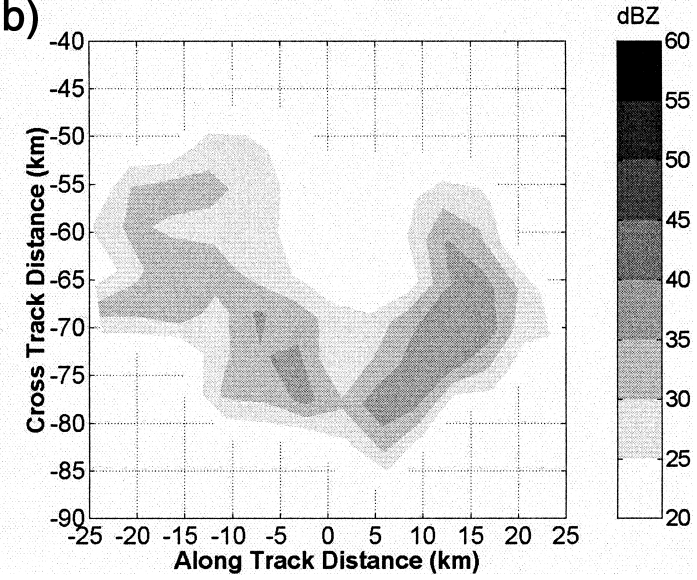

FIG. 9. Matched resolution volume and aligned, reflectivity data at 4-km CAPPI for TEFLUN-B data on 13 Aug 1998: (a) GR reflectivity with PR beams A and B shown, and (b) PR reflectivity.

effect on measurements could be equal to the measurement uncertainty in magnitude. It is logical then to ask whether or not this effect is truly seen in real data. Radar observations were collected during the TRMM TEFLUN-B field campaign, which are used to validate the proposed procedure for aligning SR with GR measurements.

\section{Alignment example}

An example of the polynomial technique to correct for geometric warping is applied to TEFLUN-B data taken on 13 August 1998 using TRMM PR and GR data. A polynomial fit was determined between pointpairs found for PR and GR images. For this example the $\alpha$ coefficients (relative to the storm cell center) were found to be $\mathbf{A}=[-0.3809,0.9895,-0.0049,0.0039$, $0.0010,0.0032]^{\mathrm{T}}$ and the $\beta$ coefficients were found to be $\mathbf{B}=[-0.2676,-0.0097,0.9704,-0.0012$, $-0.0006,-0.0000]^{\mathrm{T}}$, indicating a shift in the PR image (relative to GR) in the along- and cross-track directions, possibly some rotation (or slight scaling), and very little

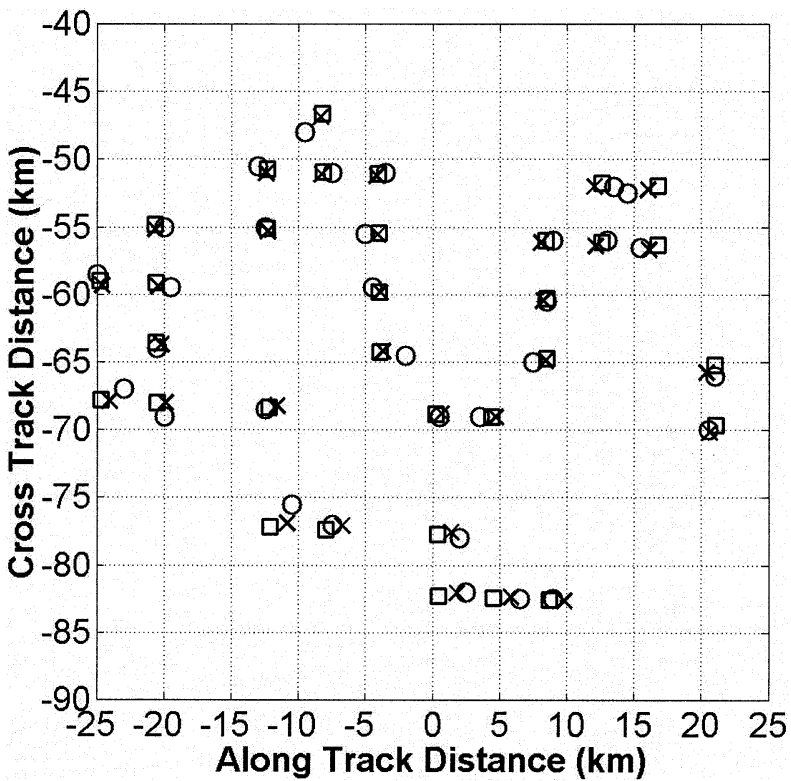

FIG. 10. PR and S-POL contour edge point-pairs at 4-km CAPPI after resolution volume matching.

other distortion effects. The relative shift in the vertical direction was also determined at each of these altitudes and the average was found to be $0.0212 \mathrm{~km}$.

CAPPI images of GR and PR measurements at $4 \mathrm{~km}$, aligned and matched in resolution volume, are shown in Figs. 9a and 9b, respectively. Two PR beams (A and B) that pass through the storm cell are indicated by circles shown in Fig. 9a drawn to scale. A plot of PR and GR point pairs at the 4-km altitude level is shown in Fig. 10 with circles denoting GR edge points, squares denoting PR edge points before alignment and $x$ 's denoting PR edge points after alignment. Note that after alignment, PR edge points are more closely aligned with GR edge points. The statistics before and after alignment of the spatial location between the edge points of the two radars, in terms of the bias and root-mean-square error (rmse), are computed for the $x$ and $y$ coordinates separately. These statistics, for $M$ points, are defined for the $x$ coordinates as follows:

$$
\begin{aligned}
\operatorname{bias}_{x \text { coord }} & =\frac{1}{M} \sum_{k=1}^{M}\left[x(\mathrm{GR})_{k}-x(\mathrm{PR})_{k}\right] \\
\text { rmse }_{x \text { coord }} & =\left\{\frac{1}{M} \sum_{k=1}^{M}\left[x(\mathrm{GR})_{k}-x(\mathrm{PR})_{k}\right]^{2}\right\}^{1 / 2},
\end{aligned}
$$

where $x(\mathrm{GR})$ and $x(\mathrm{PR})$ are the set of $x$ coordinates for GR and PR, respectively. Similar expressions are also used for the set of $y$-coordinate points. The Euclidean norm is taken of the statistics found for the $x$ and $y$ coordinates from which the final statistics are derived. A comparison of the final statistics before and after PR mapping shows improvement in the alignment between GR and PR datasets (i.e., minimization of the geometric distortion in PR observations relative to GR) - the bias 
(a)

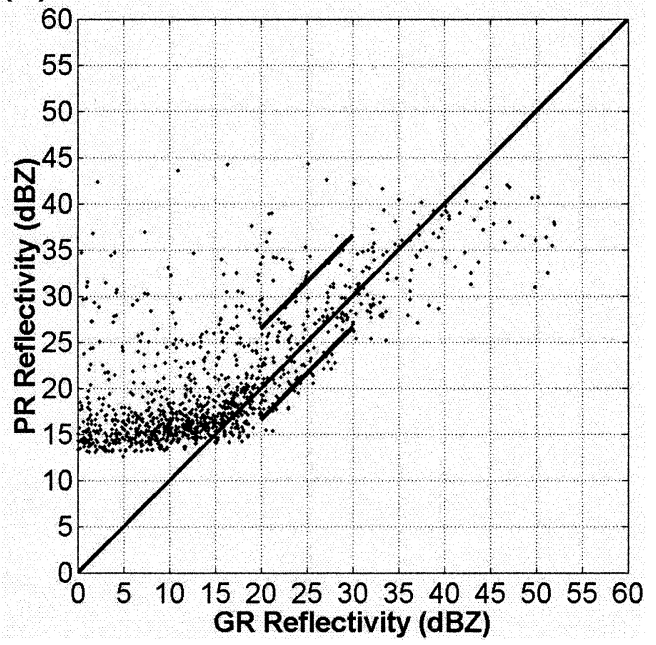

(c)

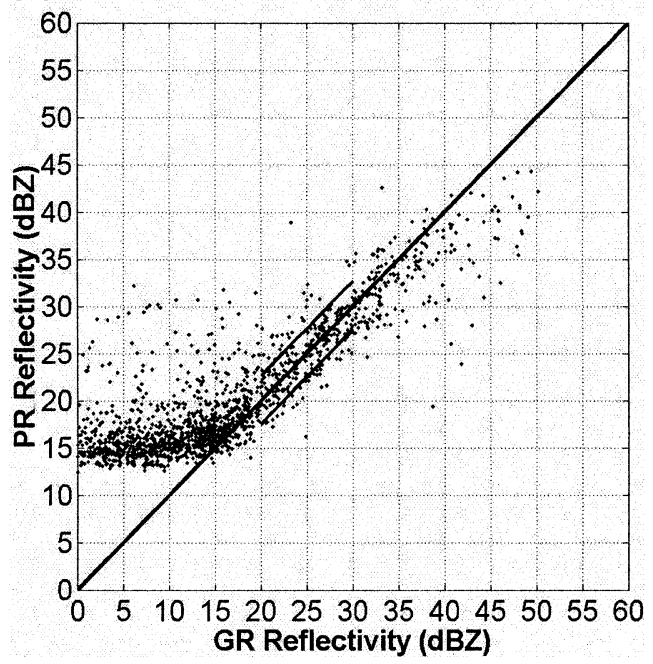

is seen to decrease from 0.2360 to 0.0509 , while the rmse decreases from 1.1911 to 0.9585 . Additionally, the area of PR reflectivity equal to, or above, $20 \mathrm{dBZ}$ is seen to be about $2.2 \%$ larger than the area of GR reflectivity, for the same contour, before alignment and about $2.0 \%$ after. Note that the percentage before alignment is approximately the same as the model developed using CHILL radar to simulate PR warping.

While the above analysis validates the alignment procedure in a spatial sense, the primary objective is the alignment of measurements so that meaningful and accurate validation of spaceborne meteorological algorithms can be conducted. Attenuation affects on PR return echoes, and the difference in receiver noise floor limits of GR and PR, make it difficult to statistically quantify the alignment procedure for all reflectivity levels. Nonetheless, it can be seen from the reflectivity scattergrams between SPOL and PR measurements shown in Figs. 11a-c that there is good improvement (b)

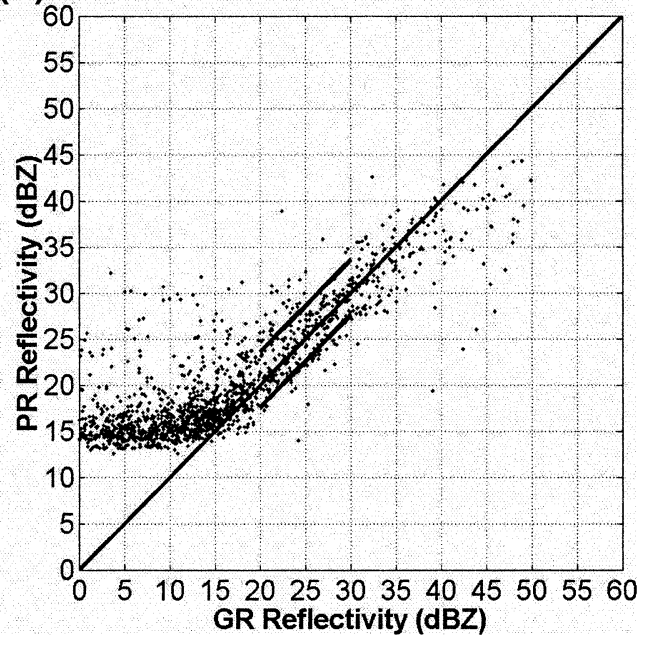

FIG. 11. Scattergrams of GR and PR reflectivity data (a) without volume matching or geometric correction; (b) with volume matching, but without geometric correction; and (c) when both GR and PR are matched in volume and PR geometric distortion is considered.

when resolution volume matching and distortion effects are considered. Figure 11a shows the scattergram of reflectivity between GR (i.e., SPOL) and PR without volume matching or distortion correction, while Fig. 11b shows the scattergram when only volume matching is considered, but not the effects of PR geometric distortion. Figure 11c shows the scattergram after the GR and PR datasets have been matched in resolution and the effects of geometric distortion on PR have been minimized. For reflectivities between 20 and $30 \mathrm{dBZ}$, which is in a range above the PR noise floor and where the effects of PR attenuation are assumed insignificant (and, also for reflectivity values above $2 \mathrm{~km}$ in altitude to reduce the effects of surface clutter return in PR measurements), it can be seen that the normalized standard error (NSE) between GR and PR reflectivity measurements decreases as volume matching and distortion effects are considered. The NSE is defined, for $N$ number of data points, as 


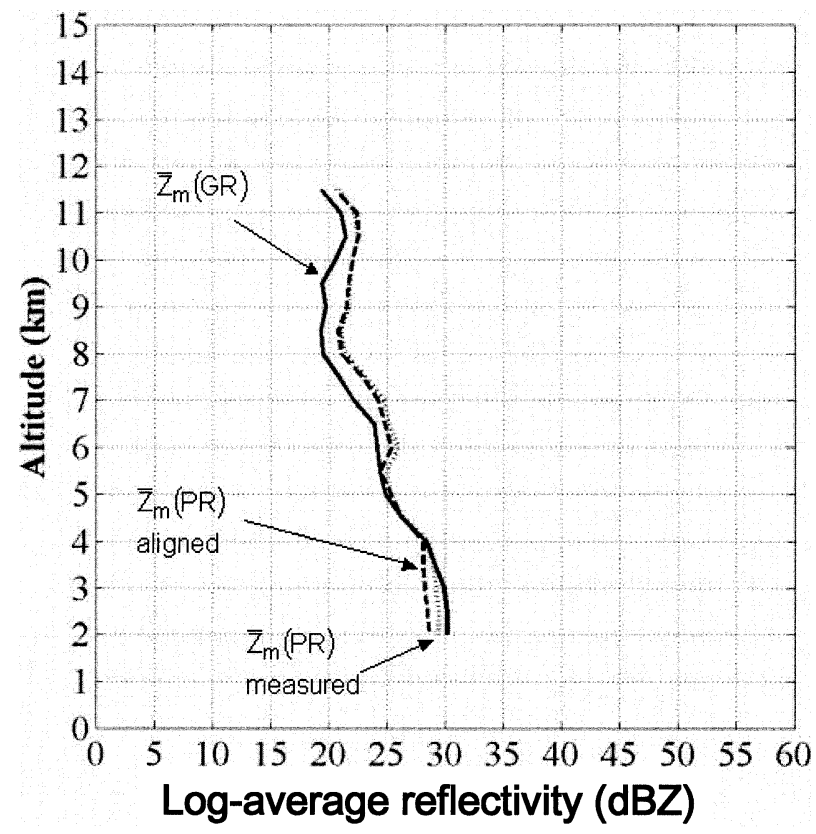

FIG. 12. Plot of average $Z_{h}$ vs altitude for log-averaged reflectivity.

$\mathrm{NSE}=\frac{\left\{\frac{1}{N} \sum_{n=1}^{N}\left[Z_{m}(\mathrm{GR})_{n}-Z_{m}(\mathrm{PR})_{n}-b\right]^{2}\right\}^{1 / 2}}{\bar{Z}_{m}(\mathrm{GR})}$,

where $b=\bar{Z}_{m}(\mathrm{GR})-\bar{Z}_{m}(\mathrm{PR})$ with $Z_{m}(\mathrm{GR})$ being the measured GR reflectivity, $\bar{Z}_{m}(\mathrm{GR})$ the average GR reflectivity, $Z_{m}(\mathrm{PR})$ the measured PR reflectivity, and $\bar{Z}_{m}(\mathrm{PR})$ the average PR reflectivity. The NSE is com- puted to be $20.36 \%, 12.09 \%$, and $10.35 \%$ for the following cases: (i) without volume matching or geometric correction; (ii) volume matching, but without geometric correction; and (iii) when both GR and PR are matched in resolution volume and PR geometric distortion is considered, corresponding to the scattergrams in Figs. 7a, $7 \mathrm{~b}$, and $7 \mathrm{c}$, respectively. Furthermore, the correlation coefficient between GR and PR reflectivity measurements, which can be defined by

$$
\text { coef }=\frac{\frac{1}{N} \sum_{n=1}^{N}\left[Z_{m}(\mathrm{GR})_{n}-\bar{Z}_{m}(\mathrm{GR})_{n}\right]\left[Z_{m}(\mathrm{PR})_{n}-\bar{Z}_{m}(\mathrm{PR})_{n}\right]}{\sigma\left[Z_{m}(\mathrm{GR})\right] \sigma\left[Z_{m}(\mathrm{PR})\right]}
$$

where $\sigma$ is the standard deviation of the quantity, and is found to increase from 0.3778 to 0.5728 and to 0.6801 for each of the cases i, ii, and iii, respectively.

The standard deviation of reflectivity between the two radars for each case are indicated by the solid lines in the 20-30-dBZ GR reflectivity interval in Figs. 11a-c. The average difference between GR and PR (i.e., GR - PR) in this interval is found to be $-1.57 \mathrm{~dB}$ with standard deviation of $4.95 \mathrm{~dB}$ when volume matching and geometric correction are not performed. After volume matching, the average difference and standard deviation were found to be -0.61 and $3.00 \mathrm{~dB}$, respectively. These values become -0.14 and $2.57 \mathrm{~dB}$ when geometric distortion correction is also applied after resolution volume matching has been performed. If measurement uncertainty in GR and PR instruments is as- sumed to be $1 \mathrm{~dB}$, then the expected standard deviation in the scatterplots about their average difference should be no more than $2 \mathrm{~dB}$. The amount of variability beyond this could be due to time synchronization mismatch between datasets, attenuation effects on PR return signal (even in the 20-30-dBZ interval), or residual errors in alignment and resolution volume matching. In the last case, it is seen that when resolution volume matching is performed the standard deviation is significantly reduced (nearly $2 \mathrm{~dB}$ ). Likewise, after geometric distortion correction is applied the standard deviation about the average difference is further reduced.

A plot of log-averaged reflectivities across a horizontal plane as a function of altitude is shown for the 13 August dataset in Fig. 12. Both the PR measured and aligned reflectivities are shown (measured indicated 
(a)

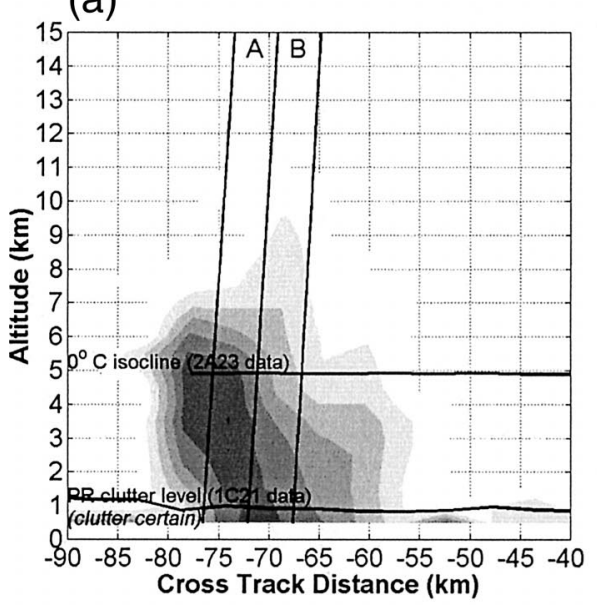

(c)

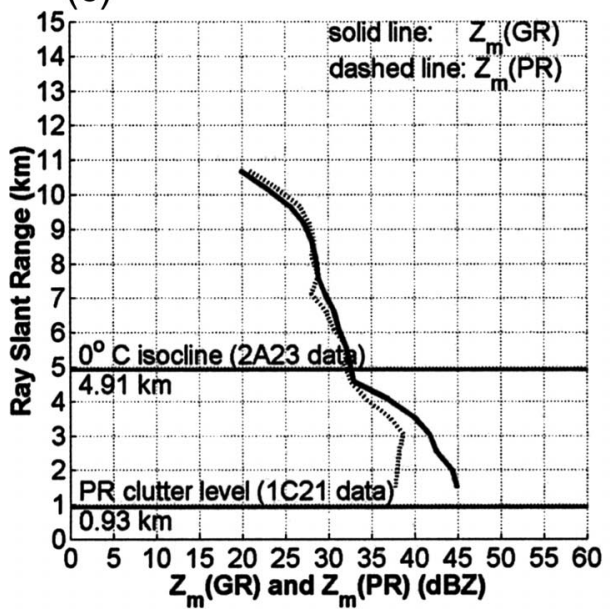

$\mathrm{dBZ}$

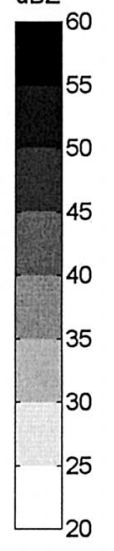

20

FIG. 13. TEFLUN-B data on 13 Aug 1998: (a) vertical cross section of GR reflectivity image (volume matched and aligned) indicating PR beams A and B shown by solid lines and drawn to scale, (b) plot of PR and GR reflectivity vs ray slant range for beam A, and (c) plot of PR and GR reflectivity vs ray slant range for beam $B$.

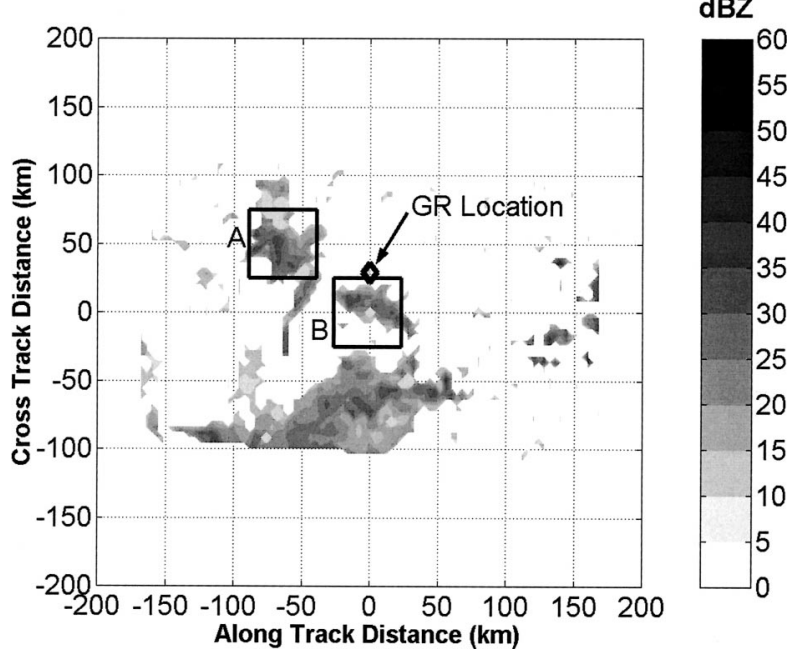

FIG. 14. Kwajalein PR reflectivity data on 10 Jul 2000 at 2-km CAPPI. (b)

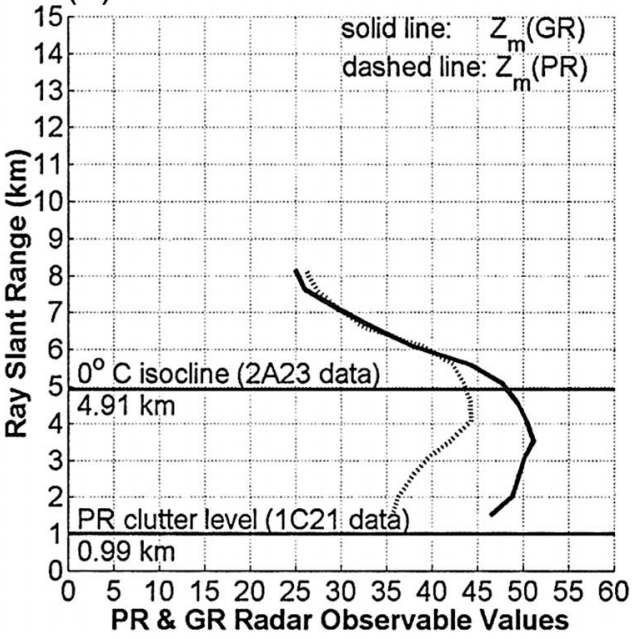

PR \& GR Radar Observable Values

by the dotted line and aligned by the dashed line) along with log-averaged GR reflectivity, shown as a solid line [where $Z_{m}(\mathrm{GR})$ is used to denote ground radar measured reflectivity and $Z_{m}(\mathrm{PR})$ TRMM PR reflectivity]. The plot indicates the presence of geometric warping in the PR data taken during the TEFLUN-B experiment similar to the results of the simulation of PR data presented earlier. (Note that the reflectivity values are averaged in log scale.) At lower altitudes, where there are nonuniform regions of reflectivity, warping is seen to increase the PR reflectivity observations by as much as $1.0 \mathrm{~dB}$. At higher altitude where attenuation is low, the bias between GR and PR is about $-1.5 \mathrm{~dB}$. This result in bias is consistent with that reported by Anagnostou et al. (2001) who found a $-1.3 \mathrm{~dB}$ difference between GR and PR for TRMM-LBA data.

After resolution volume matching and geometric distortion correction, direct point-to-point comparisons between GR and PR can be made. A cross section of the reflectivity profile for GR data is shown in Fig. 13a with PR beams A and B indicated by the solid lines drawn to scale. The plot of PR and GR reflectivities along beam 
(a)

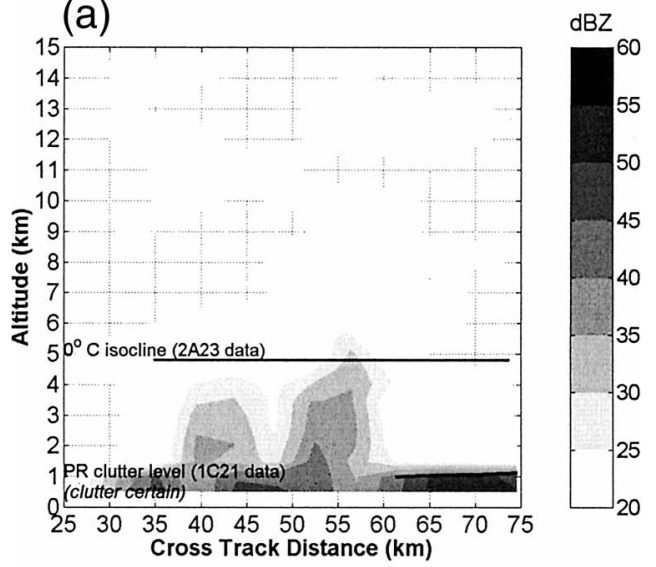

(b)

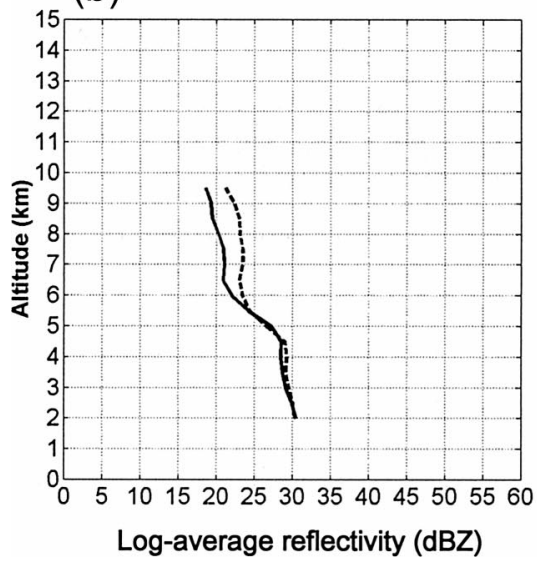

FIG. 15. Kwajalein data region A: (a) PR cross-section reflectivity image through the most intense part of the storm cell, and (b) logaveraged reflectivity vs altitude for GR reflectivity, and PR reflectivity before and after alignment.

$\mathrm{A}$ is shown in Fig. 13b, and along beam B in Fig. 13c. In both along-beam plots, the bias between GR and PR is, again, within expected values. In fact, in both plots, the vertical profile of GR and PR agree well above the freezing level (which is indicated by the solid line taken from PR 2A23 data).

Finally, a case is presented in which the difference between GR and PR reflectivity measurements is significant (on the order of a few decibels). Data were collected simultaneously from the Kwajalein S-band radar and PR on 10 July 2000. Precipitation radar data sampled at 4-km horizontal resolution at 2-km CAPPI along the TRMM ground track are shown in Fig. 14. Two storm cells were selected for analysis from the dataset, labeled A and B, and marked by the squares as shown in Fig. 14 along with the location of the Kwajalein ground radar (denoted as GR in the figure). Resolution volume matching and alignment was performed for each of the regions. A cross section of PR reflectivity through the most intense part of storm cell A is shown in Fig. 15a, and a plot of the log-averaged reflectivity (a)

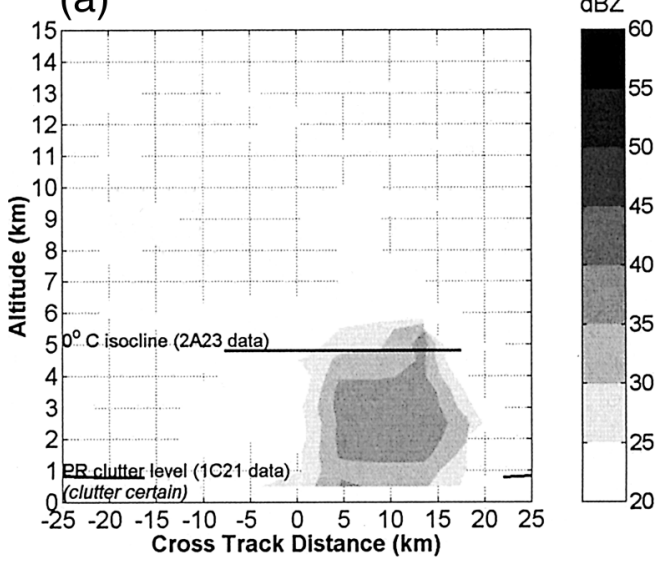

(b)

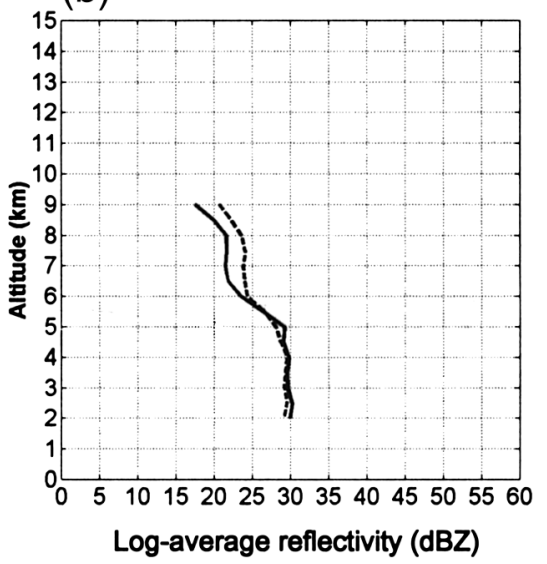

FIG. 16. Kwajalein data region B: (a) PR cross-section reflectivity image through most intense part of the storm cell, and (b) $\log$-averaged reflectivity vs altitude for GR reflectivity, and PR reflectivity before and after alignment.

across a horizontal plane versus altitude for GR (solid line) and PR reflectivity after correction (dashed line) was constructed, which is shown in Fig. 15b. The average difference between GR reflectivity and PR reflectivity after correction is about $-2.75 \mathrm{~dB}$ between the 6.5- and 9.5-km altitude levels (which is above the isocline level and roughly below the cloud top).

Similarly, the cross section of PR reflectivity through the most intense part of storm cell B is shown in Fig. 16a. The log-averaged plots of GR reflectivity and PR reflectivity after correction is shown in Fig. 16b. The average difference between GR and PR is about -2.25 $\mathrm{dB}$ in the altitude levels between 6.5 and $9.0 \mathrm{~km}$. Note that these storm cells are at different distances from the ground radar location $(80 \mathrm{~km}$ for cell $\mathrm{A}$ and $28 \mathrm{~km}$ for cell B), and at different distances from the TRMM ground track $(50 \mathrm{~km}$ for cell $\mathrm{A}$ and nearly coincident with the TRMM ground track for cell B). However, the difference between GR reflectivity and PR aligned reflectivity is nearly the same for both cases (within 0.5 $\mathrm{dB})$. This simple comparison indicates the robustness 
of the method even when there exists significant differences between GR and SR measurements as well as when measurements are made at different distances from the radars.

\section{Summary}

A method to align radar measurements from two different platforms (i.e., a static terrestrial and a moving space-based platform) has been developed based on variable resolution volume matching and a polynomial fit to low-intensity contour images between the two radars. Though this method was developed for matching data between a spaceborne radar system and a ground-based system, the method could also be applied to any two radars: ground-ground, space-space, airborne-ground, space-airborne, etc., as well. The method takes into account geometric distortion induced on radar measurements if there is relative movement between the two platforms. An example using real data was used to demonstrate the matching procedure between a spaceborne radar and ground-based radar. Simulation was performed on CSU CHILL radar reflectivities to model PR return. It was found that under certain circumstances geometric warping can cause nearly a $1-\mathrm{dB}$ offset from the true reflectivity field. This effect was also seen in data at altitudes where reflectivity was nonhomogeneous. Finally, plots of GR and PR reflectivity along the PR were shown as an example of the type of direct comparison that can be made between space and ground radar measurements based on this technique.

Acknowledgments. This work was supported by the NASA Tropical Rainfall Measuring Mission (TRMM) Program.

\section{REFERENCES}

Anagnostou, E., C. Morales, and T. Dinku, 2001: The use of TRMM precipitation radar observations in determining ground radar calibration biases. J. Atmos. Oceanic Technol., 18, 616-628.

Bolen, S., and V. Chandrasekar, 1999: Comparison of satellite-based and ground-based radar observations of precipitation. 29th Int. Conf. on Radar Meteorology, Montreal, QC, Canada, 751-753.

$\ldots$, and —_, 2000a: Ground and satellite-based radar observation comparisons: Propagation of space-based radar signals. IEEE IGARSS 2000 Conf., Honolulu, HI, IEEE.

$\longrightarrow$, and $-2000 \mathrm{~b}$ : Quantitative cross-validation of space-based and ground-based radar observations. J. Appl. Meteor., 39, 2071-2079.

Brogan, W., 1985: Modern Control Theory. Prentice Hall, 736 pp.

Meneghini, R., and T. Kozu, 1990: Spaceborne Weather Radar. Artech House, 208 pp.

NASA, 1999: Tropical Rainfall Measuring Mission Science Data and Information System: Interface Control Specification (ICS) between the Tropical Rainfall Measuring Mission Science Data and Information System (TSDIS) and the TSDIS Science User (TSU). NASA GSFC Doc. TSDIS-P907, Vol. 3, Release 4.04. [Available online at http://tsdis.gsfc.nasa.gov/tsdis/Document/ ICSVol3.pdf.]

Schowengerdt, R., 1997: Remote Sensing: Models and Methods for Image Processing. 2d ed. Academic Press, 572 pp. 